\title{
TEMPORAL DYNAMICS IN THE STRUCTURE AND COMPOSITION OF A DESERT RODENT COMMUNITY
}

\author{
Katherine M. Thibault, ${ }^{1}$ Ethan P. White, and S. K. Morgan Ernest \\ Department of Biology, University of New Mexico, Albuquerque, New Mexico 87131 USA
}

\begin{abstract}
The rank-abundance distribution (RAD) represents the manner in which species divide resources. Community-specific division rules that determine resource allocation among species, and thereby the shape of the RAD, have been hypothesized to account for observed stability of local species richness over time. While the shape of the RAD has been well studied, the temporal dynamics of this distribution have received much less attention. Here we assess changes in the shape of the RAD through time in a desert rodent community in Arizona (USA). Because energy use may be more appropriate for studying resource division than abundance, we also evaluate an energetic equivalent of the RAD. Significant, directional trends in the shapes of both distributions are present in this community. These changes are driven by trends in the relative abundances (or energy use) of Ranks 2, 3, and 4, and are significantly correlated with variation in total energy use by the community and with compositional change. Our results suggest that (1) rank-abundance and rank-energy distributions are not static and can change directionally through time, (2) species richness and rank distributions are not necessarily as intimately connected as early studies assumed, and (3) rank-abundance and rank-energy distributions are influenced by both the amount of energy available to the community and species-specific interactions.
\end{abstract}

Key words: community properties; community structure; desert rodents; Portal, Arizona, USA, desert rodent community; rank-abundance distribution; rank-energy distribution; resource allocation; species richness; temporal dynamics.

\section{INTRODUCTION}

Understanding how resources are divided among species can elucidate the mechanisms of coexistence within communities, and thus those underlying local species richness. Traditionally, resource division has been studied using the rank-abundance distribution (RAD; e.g., MacArthur 1957, Whittaker 1965, Hubbell 1979), which quantifies the relative abundances of species. This distribution of abundance is assumed to reflect the underlying pattern of resource allocation among species and to provide insight into the processes influencing coexistence (Tokeshi 1999, Sugihara et al. 2003). Analyses of RADs typically attempt to determine mechanisms of community assembly via two methods: (1) statistical description and theoretical derivation of observed distributions (e.g., MacArthur 1957, Preston 1962, May 1975, Sugihara 1980, Tokeshi 1990, Hubbell 2001) and (2) comparison of distributions from communities with varying species richness and environmental conditions (e.g., Whittaker 1965, Bazzaz 1975, Hubbell 1979, Kempton 1979). While

Manuscript received 12 February 2004; revised 4 May 2004; accepted 25 May 2004. Corresponding Editor: M. S. Boyce.

${ }^{1}$ E-mail: katet@unm.edu these approaches have yielded valuable insights into the various shapes of RADs, there is still no consensus regarding the mechanistic processes underlying the distribution.

The RAD is typically assumed to be a static descriptor of community structure (e.g., MacArthur 1957, Tokeshi 1990) and, as a result, is often calculated from either a single year of data or from data pooled across several years for a single site (e.g., Preston 1948, Hubbell 1979, McGowan and Walker 1985). While some features of communities are resistant to change (e.g., total abundance, biomass, energy flux, species richness), others (e.g., species composition) are highly dynamic (e.g., Margalef 1968, May 1973, Hughes and Roughgarden 1998, Ives et al. 1999, Ernest and Brown $2001 a, b)$. Because the RAD is likely influenced by both species-specific interactions and community-level limitations, its location on the continuum between static community properties and dynamic species composition is unclear. This uncertainty remains, in part, because the dynamics of the RAD have never been quantitatively assessed. Understanding how the RAD changes through time will provide important insights into the processes generating the distribution, elucidating how species-specific interactions and commu- 


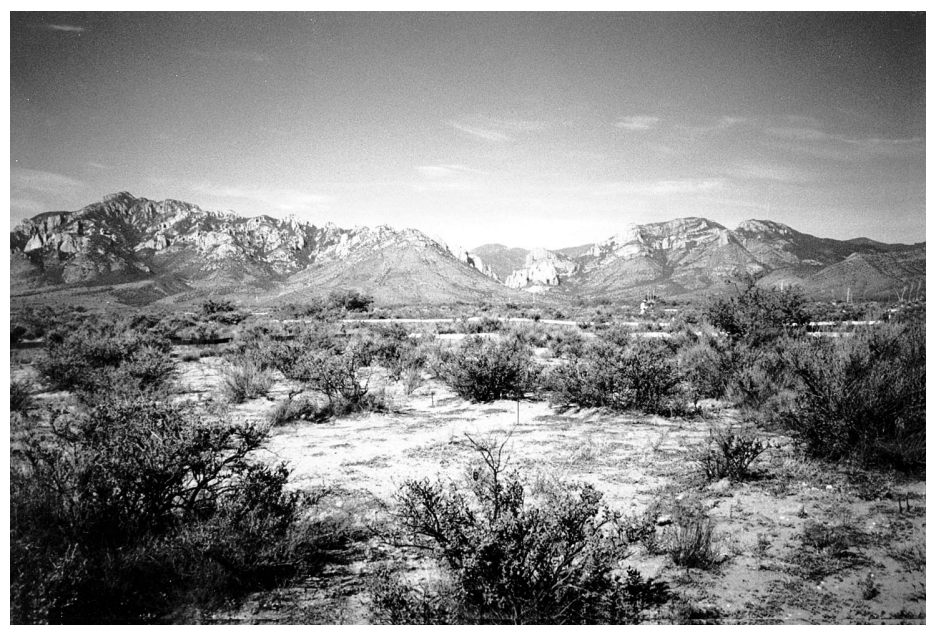

Plate 1. The Portal Long Term Research in Ecological Biodiversity site in southeastern Arizona, USA, where the interactions among desert rodents, ants, and annuals have been experimentally studied since 1977 (note the fencing around the study plots which controls rodent access). Note also the currently high density of shrubs in the study area that is likely involved in the reorganization of the desert rodent community. Photo credit: S. K. Morgan Ernest.

nity-level constraints combine to influence the general shape of the distribution. In addition, evaluation of the temporal dynamics of the RAD will allow for testing the idea that a consistent pattern of resource allocation, a general division rule, serves to maintain species richness through time (Brown et al. 2001).

Further insights into the division of resources may also be gained from examining the distribution of energy use, in addition to abundance, among species. Abundance and energy use can yield different results, because organisms of different size have different metabolic rates (Pagel et al. 1991, Taper and Marquet 1996, Marquet et al. 2003). As a result, energy flux likely provides a more accurate reflection of resource use than abundance (Harvey and Godfray 1987, Pagel et al. 1991). While frequency distributions of energy use have been published, to our knowledge the energetic equivalent of the RAD has not.

Here we examine the temporal dynamics of resource division, as represented by the RAD and the rank-energy distribution (RED), using long-term data on a granivorous rodent community. The species richness and total energy use of this community have remained relatively constant over 25 years (Brown et al. 2001, Ernest and Brown 2001a), whereas species composition has changed significantly through time (Valone and Brown 1995, Ernest and Brown 2001b). This allows us to evaluate the dynamics of resource allocation and to assess the relative roles of energetic constraints and species interactions.

\section{Methods}

We assessed changes in the composition and community structure of the desert rodent community at a long-term site established by J. H. Brown and associates in 1977. The site, located near Portal, Arizona, USA $\left(31.9^{\circ} \mathrm{N}, 109.1^{\circ} \mathrm{W}\right)$ at an elevation of $1330 \mathrm{~m}$, is a mixture of Chihuahuan Desert shrubland and arid grassland (see Plate 1). Within the site there are 24 0.25 -ha experimental plots. On these plots rodents have been censused monthly since 1977 . We used the aggregated data from 10 unmanipulated control plots for our analysis. We restricted our analysis to the 14 rodent species that are predominantly granivorous in order to ensure that they subdivide a common resource: (i.e., Baiomys taylori, Dipodomys merriami, D. ordii, D. spectabilis, Chaetodipus baileyi, C. hispidus, C. intermedius, C. penicillatus, Perognathus flavus, Peromyscus eremicus, $P$. maniculatus, Reithrodontomys megalotis, $R$. montanus, and $R$. fulvescens (Ernest and Brown 2001a). See Brown (1998) for further details on methodology at the site.

To assess the directionality of trends in composition and community structure through time, we modified a method recently proposed by Collins, Micheli, and Hartt (2000; henceforth CMH) (see also Collins 2000). This approach is based on using the Euclidean Distance (ED) to measure the difference in composition and structure between two communities. The ED represents the distance between two points in multidimensional space. In the case of composition,

$$
\mathrm{ED}=\sqrt{\left(a_{1}-a_{2}\right)^{2}+\left(b_{1}-b_{2}\right)^{2}+\ldots\left(n_{1}-n_{2}\right)^{2}}
$$

where $a_{1}$ through $n_{1}$ are the mean relative abundance values of different species during some year, and $a_{2}$ through $n_{2}$ are the mean relative abundance values of the same species during another year. The ED measures the difference in community composition between two points in time. We first calculated the ED of the community composition between every possible pair of years in the time series. In the $\mathrm{CMH}$ approach, all ED values are then regressed against the square root of their time lags, thereby quantifying the difference in composition as a function of their temporal separation. However, this type of analysis suffers from inflated sample size (in our case 300 points are generated from 25 samples) and lack of independence among points (as many as $24 \mathrm{ED}$ values share a single community). Consequently, the interpretation of the variance and hence the statistical significance of the relationship is 

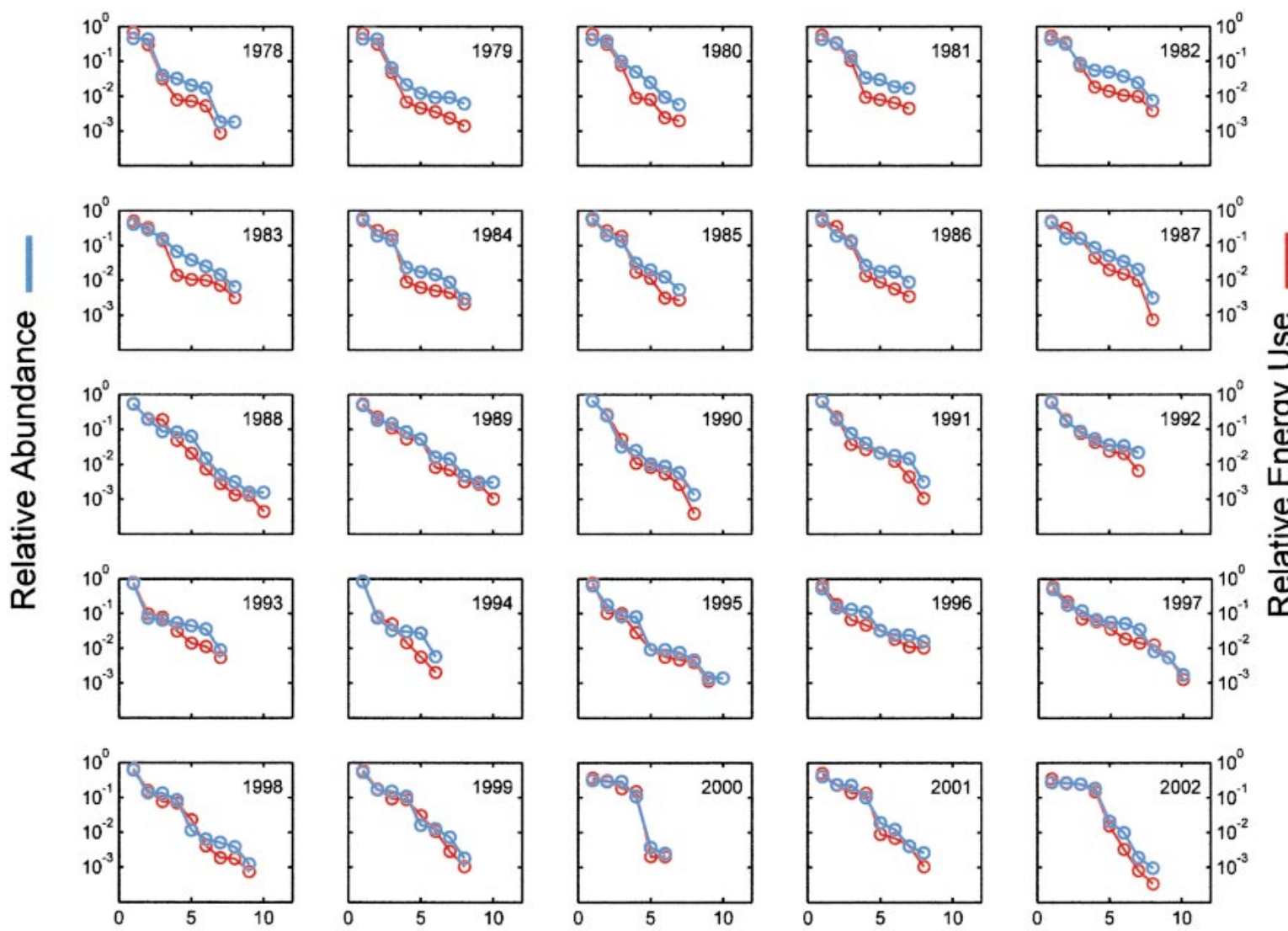

\section{Species Rank}

FIG. 1. Annual rank-abundance (blue) and rank-energy (red) distributions for the granivorous rodent community at J. H. Brown's long-term study site near Portal, Arizona, USA, from 1978 through 2002. The $y$-axes are logarithmically scaled. Changes in the shape of both distributions through time are evident, suggesting that, despite relative constancy of species richness, the allocation of resources among species is dynamic.

problematic. To assess statistical significance of the CMH slope, we generated 10000 randomized data sets in which communities were randomly ordered with respect to time, by permuting the columns of the yearby-species matrix. We then compared the distribution of $\mathrm{CMH}$ slopes for the randomized data to the $\mathrm{CMH}$ slope for the observed data. The probability for the test of equality between the random and observed slopes was determined by dividing the number of instances where the random slope was greater than the observed slope by 10000 . This procedure is similar to that of the Mantel test (Legendre and Legendre 1998), and both tests yielded comparable results.

We used a similar technique to evaluate changes in the rank-abundance distributions (RAD) and rank-energy distributions (RED) over the course of the study. To generate the REDs, we calculated the energy use of each species by summing the energy use of all individuals of that species estimated from the allometry of field metabolic rates (Energy use $=5.69[\text { Mass }]^{0.75} ; r^{2}$ = 0.99; data from Nagy et al. [1999]; see White et al., [in press] for further details). This approach is an im- provement on that previously used for generating energy distributions (Harvey and Godfray 1987, Pagel et al. 1991), because it does not rely on any specific functional relationship between mass and abundance. For both rank-energy and rank-abundance distributions, we compared years using

$$
\mathrm{ED}=\sqrt{\left(\hat{A}_{1}-\hat{A}_{2}\right)^{2}+\left(\hat{B}_{1}-\hat{B}_{2}\right)^{2}+\ldots\left(\hat{N}_{1}-\hat{N}_{2}\right)^{2}}
$$

where $\hat{A}_{1}$ through $\hat{N}_{1}$ were the standardized mean relative abundance or energy values of ranks $A$ through $N$ during some year, and $\hat{A}_{2}$ through $\hat{N}_{2}$ were the standardized mean relative abundance or energy values of ranks $A$ through $N$ during another year. The relative abundance or relative energy values were standardized within each rank to have mean 0 and standard deviation 1. This was done so that all ranks would have equal weights in the analysis and thus allow us to quantify changes in the overall shape of the distributions. The standardization is achieved by defining the standardized $A_{i}$ as $\hat{A}_{i}=\left(A_{i}-\bar{A}\right) / \sigma_{A}$, where $A_{i}$ is the relative abundance of rank $A$ in the focal year, $\bar{A}$ is the mean relative abundance of that rank across all years, and $\sigma_{A}$ 
is its standard deviation. We used the same randomization approach that we used for the composition data, but due to this standardization, these slopes are not directly comparable with the slope for composition.

We also conducted both sets of analyses using the original $\mathrm{CMH}$ approach and a simple comparison of the initial year of the study to all subsequent years. The results of both approaches were qualitatively similar. For the RAD we did not use parameters of standard theoretical or empirical models (see Tokeshi [1999] for a summary of popular models), because we found them to be primarily influenced by the dominant species and therefore inadequate to evaluate changes in the shape of the distribution as a whole. Our approach relies only on the observed data and does not make any assumptions about the shape of the distribution. To determine in which ranks of the RAD and RED changes occurred, we constructed time series of the standardized relative abundances and energy use of each rank and evaluated trends using ordinary least-squares regression (we recognize that autocorrelation compromises the assessment of statistical significance).

To evaluate the relative contributions of changes in composition and changes in resource availability to the observed changes in the rank-abundance and rank-energy distributions, we calculated the ED between total energy use for all possible pairs of years (because total energy use is a single variable, this is equivalent to taking the absolute value of the difference between the energy use of the community at some time and the energy use of the community at another time). Energy use of the community in each year was calculated by summing the energy use of the individual species as calculated above. The composition ED and the energy ED for each pair of years were then used in a multiple regression to predict the RAD and the RED Euclidean distances.

\section{REsults}

Changes in the form of the annual rank-abundance distribution (RAD) and the rank-energy distribution (RED) through time are readily apparent (Fig. 1). While general similarities between the shapes of the RAD and RED are evident, there were substantial differences between the distributions. These differences were more striking during the initial years of the study, presumably due to the greater mass differences among the dominant species that result from the predominance of the relatively large Dipodomys spectabilis (mean mass $=120.2 \mathrm{~g}$ ) only during the early years of the study.

Euclidean distances (EDs) between annual species composition, RADs, and REDs generally increased with time lag (Fig. 2A-C). Randomization analyses of temporal trends in EDs reveal significant directional changes in both the composition and the structure of the community, with the slopes of the $\mathrm{CMH}$ analyses significantly greater for the observed data than for the randomized data (composition: slope $=0.105, P<$
0.0001 ; RAD: slope $=0.35, P=0.0018$; RED: slope $=0.36, P=0.007$; Fig. $2 \mathrm{D}-\mathrm{F})$. Thus, all three descriptors of the community are changing through time, although the shifts in species composition are more extreme (an approximately five-fold increase) than those in the rank abundance and energy distributions (an approximately two-fold increase) over the course of the study (Fig. 2A-C).

Within this granivorous rodent community, 3 of the 10 ranks have experienced significant changes in relative abundance over time. The relative abundance of Rank 2 has decreased, whereas Ranks 3 and 4 have increased their dominance (all $P<0.02$; see Appendix). Significant changes in relative energy use of Ranks 2 and 4 parallel the shifts in abundance $(P<$ 0.002; see Appendix), but the relative energy use of Rank 3 has not changed significantly. This discrepancy is likely due to the fact that the species occupying Rank 3 generally tended to be smaller in the later years of the study, effectively compensating for the increased abundance. Specifically, the principal occupant of this rank changed over time from $D$. ordii (mean mass $=48.9$ g) to Chaetodipus penicillatus (mean mass $=17.3 \mathrm{~g}$ ).

Variation in both total energy use and species composition was significantly correlated with the observed variation in the rank-abundance distribution, and the partial correlation coefficients for each variable were similar to one another $(r=0.385$ and 0.419 , respectively; $P<0.001$ ). The multiple regression explained $30 \%$ of the total variation in the rank-abundance ED. Results were similar for the rank-energy distribution, though in this case the rank-energy ED was more strongly correlated with the energy ED than the composition ED ( $r=0.38$ and 0.25 respectively; $P<$ $0.001)$. The multiple regression explained just over $20 \%$ of the total variation in the RED.

\section{DISCUSSION}

Our study shows that the pattern of resource division among species changes through time in this desert rodent community. The community exhibited a temporal trend in both the rank-abundance distribution (RAD) and the rank-energy distribution (RED) (hereafter referred to collectively as the "rank distributions"), indicating that there have been regular changes in the relative abundance or energy flux across ranks. These patterns suggest that resources have been redistributed among ranks over time, causing temporal changes in the overall shape of the distributions. This result is particularly striking given that both metrics of resource division, abundance and energy use, yield similar conclusions, despite the fact that they have often produced disparate patterns and dynamics (Pagel et al. 1991, Taper and Marquet 1996, Ernest and Brown 2001a, Marquet et al. 2003, White et al., in press).

The observed directional shifts in resource allocation among species contradict the general assumption that the RAD can be treated as a static property of a com- 

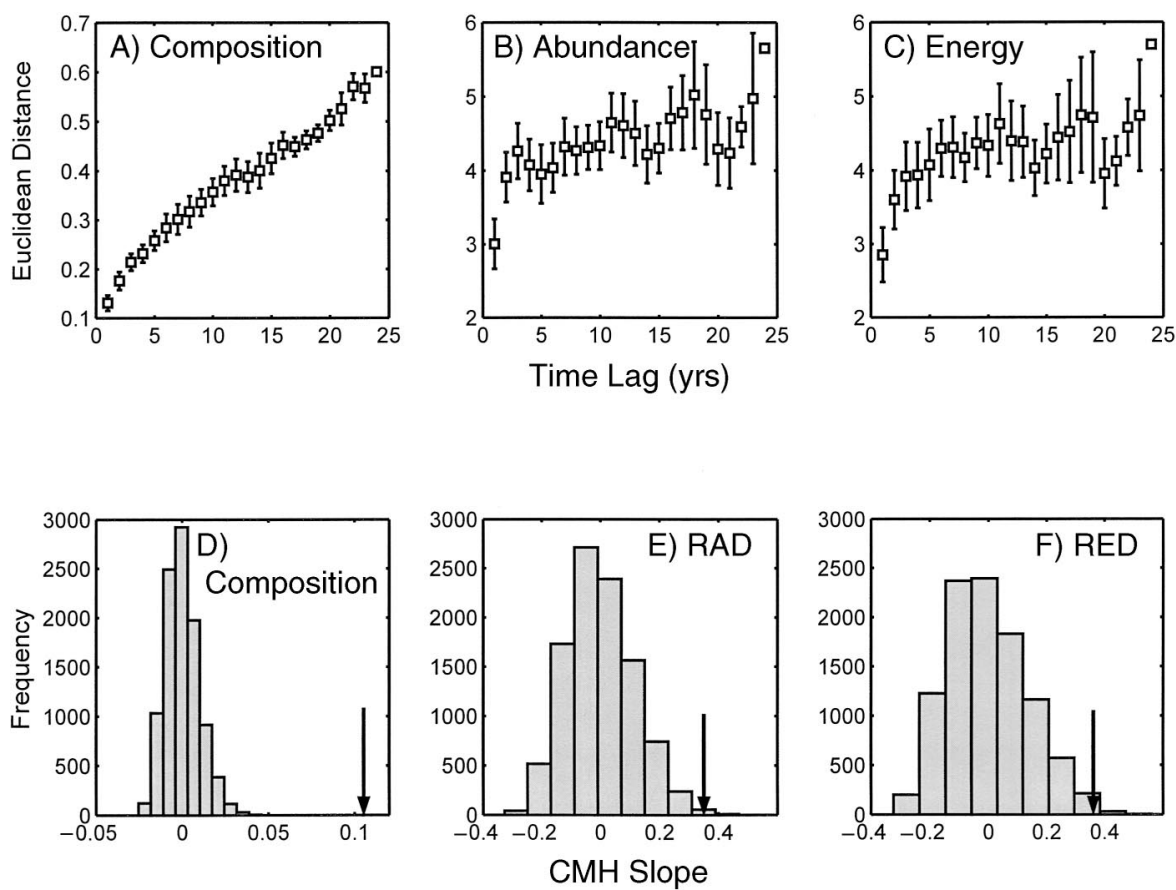

FIG. 2. Temporal trends in species composition and community structure. Euclidean distances (ED) between all possible pairs of years (1978-2002), as a function of time lag for (A) annual species composition (B), rank-abundance distributions (RAD), and (C) rank-energy distributions (RED). Data are means \pm 1 sE. Frequency distributions of slopes generated from resampling of randomized data (10000 iterations) are shown for (D) composition, (E) RAD, and (F) RED. Observed slopes obtained from the Collins et al. (2000) method (CMH) are represented by the black arrow. All of the slopes are significant (all $P<0.01$ ), indicating directional changes through time in all community properties. The strong compositional shifts that have occurred in this community (A, D), in addition to interannual variability in energy availability, have contributed significantly to the observed changes in the rank-abundance and rank-energy distributions.

munity (e.g., MacArthur 1957, Tokeshi 1990). This likely results from the fact that the majority of studies of the shape of the rank-abundance distribution are conducted over relatively short time scales, usually one or two years (e.g., Whittaker 1965, Hubbell 1979). Our results demonstrate that short-term data can be misleading, because differences that appear minor between two years may be embedded in a long-term trend (Fig. 1). When long-term data are available, most authors have tracked the ranks of individual species over space or time (e.g., MacArthur 1955, McGowan and Walker 1985, Bengtsson et al. 1997, Guo et al. 2002). In contrast, we analyzed the shapes of RADs and REDs without regard to species' identities. The concept of a community-wide division rule does not require that the same species consistently utilizes the same proportion of resources from one time period to the next, but that the same proportion of resources is used by the species of a particular rank regardless of identity (i.e., a process-oriented interpretation sensu Tokeshi 1999).

The dynamic nature of the division of resources in this community appears to reflect temporal variation in both species composition and total energy use. The strong, directional trend of increasing distance from the initial composition (Fig. 2A) presumably reflects the three-fold increase in shrub density that has oc- curred at the site since 1977 (Brown et al. 1997). Rodents are known to respond to the physiognomy of vegetation, which affects the availability of preferred foraging microhabitats (e.g., Brown et al. 1979, Bowers et al. 1987). Therefore, a change in shrub density should shift the frequency distributions of microhabitats. Such a directional shift would alter the resources available to each species, potentially changing its relative energy use, abundance, and even its presence or absence. The effect of habitat is supported by the increased abundance of shrubland-affiliated species (i.e., Chaetodipus baileyi, C. penicillatus) with a concomitant decrease in grassland-affiliated species (Dipodomys spectabilis, Perognathus flavus). Total community energy use, or resource availability, on the other hand, has fluctuated interannually in response to variation in rainfall and other factors (Ernest et al. 2000, Brown and Ernest 2002), but has exhibited no long-term directional trend (Ernest and Brown 2001a). The coupling of the strong compositional shift with the relatively stable resource supply likely accounts for the fact that the temporal trends in Euclidean distances of the rank distributions are not as strong as that of species composition (Fig. 2). However, further studies designed to separate the effects of species, habitat, and 
resources are required to definitively address these issues.

Despite the overall change in the shapes of the rank distributions, most ranks have used approximately the same proportion of resources through time. The changes in the shapes were driven by shifts in the abundance or energy use of only the second, third, and fourth ranks. Within the context of niche apportionment (sensu Tokeshi 1999), the species that occupied Ranks 3 and 4 progressively procured more of the niche space of the second-ranked species. These changes among the ranks undoubtedly reflect the underlying shift in the species composition of the community over this time span. In this community, D. merriami has been the most dominant species in 23 of the past 25 years, with the other two species of kangaroo rats, D. spectabilis and $D$. ordii, typically occupying the second and third ranks. However, since the local extinction of $D$. spectabilis in the early 1990 s and the colonization of the site by $C$. baileyi in 1995, C. baileyi and C. penicillatus have increased in abundance markedly, thereby appropriating Ranks two and three. Concurrently, the abundance of D. merriami has declined (Fig. A1), and $D$. ordii has taken over the fourth rank, primarily displacing the granivorous murids, Peromyscus eremicus and Reithrodontomys megalotis. As a result, the relative abundances of the top four ranks have been converging over time, yielding the strikingly different rank distributions of the latter years (Fig. 1). The turnover of species within all ranks but the first has been high (i.e., 5-8 species per rank) over the period of the study. We suspect that the fact that these particular ranks changed through time is due to the nature of the observed environmental changes and the associated species' responses. We expect that in other communities the individual ranks driving changes in rank distributions through time will depend upon the environment and the biology of the individual species. Furthermore, long-term stability in the allocation of resources among species may yet be found within communities inhabiting more constant environments.

The directional changes in the shape of the rank distributions appear to be contrary to predictions of a constant division rule (sensu Brown 1981, 1984). The idea that each community has a division rule that determines the relative resource use for each species has been important not only in understanding resource division, but it has also been proposed to explain the temporal stability of species richness through time or in response to perturbation (see Brown et al. [2001] and references therein). This rodent community has not exhibited directional changes in species richness (Brown et al. 2001, Ernest and Brown 2001a) despite significant habitat changes (Brown et al. 1997). The lack of evidence of a constant division rule operating in this community therefore leaves the mechanism(s) determining species richness obscure. Although energy is significantly related to broad-scale, geographic patterns of species richness (e.g., Currie 1991, Hawkins et al. 2003), our analysis of the allocation of the available energy at small scales did not reveal a strong, consistent pattern despite constancy of species richness. One potential, niche-based explanation is that, as the environment changed over the course of the study, the relative availability, but not the number, of available niches (i.e., foraging microhabitats) has changed, yielding altered patterns of resource division but no changes in richness. Thus, it is possible that the interaction of energy availability and niche availability dictates species richness at the local scale, with changes in both required to change species richness, but a change in only one to significantly affect the RAD.

Whittaker (1965:250) stated that "[dominance-diversity] relations are less lawful, orderly, and consistent than ecologists might wish". Although the lack of consensus concerning underlying mechanisms lends credence to his contention, we would argue that the use of the growing number of long-term studies to analyze temporal patterns of dominance and diversity in real systems could provide valuable insights. Our study represents one of the few such efforts, and the results suggest that (1) rank-abundance and rank-energy distributions can change directionally through time, (2) species richness and rank distributions are not necessarily as intimately connected as early studies assumed, and (3) RADs and REDs are influenced both by energy availability and by changes in species composition due to changes in habitat characteristics and other environmental features. We believe that further study of the dynamics of community structure and its connection to ecosystem ecology can contribute to the long-term goals of understanding the mechanisms underlying resource allocation and gradients of species diversity.

\section{ACKNOWLEDGMENTS}

We thank J. H. Brown, A. Ernest, A. P. Allen, J. R. Goheen, A. H. Hurlbert, and the many volunteers and assistants who have worked at Portal over the years. We also thank Mark Boyce, Steve Jenkins, and one anonymous reviewer for helpful comments on the manuscript. The Portal Project has been supported by many grants, most recently NSF LTREB grant DEB-0129298. K. M. Thibault was also supported by this grant. Additionally, E. P. White and S. K. M. Ernest were supported by an NSF Biocomplexity grant (DEB-0083422), and E. P. White and K. M. Thibault were supported by NSF Graduate Research Fellowships.

\section{Literature Cited}

Bazzaz, F. A. 1975. Plant species diversity in old-field successional ecosystems in southern Illinois. Ecology 56:485488.

Bengtsson, J., S. R. Baillie, and J. Lawton. 1997. Community variability increases with time. Oikos 78:249-256.

Bowers, M. A., D. B. Thompson, and J. H. Brown. 1987. Spatial-organization of a desert rodent community-food addition and species removal. Oecologia 72:77-82.

Brown, J. H. 1981. Two decades of homage to Santa Rosalia: toward a general theory of diversity. American Zoologist 21:877-888. 
Brown, J. H. 1984. On the relationship between the abundance and distribution of species. American Naturalist 124: 255-279.

Brown, J. H. 1998. The desert granivory experiments at Portal. Pages 71-95 in W. J. Resetarits, Jr. and J. Bernardo, editors. Experimental ecology. Oxford University Press, New York, New York, USA.

Brown, J. H., and S. K. M. Ernest. 2002. Rain and rodents: complex dynamics of desert consumers. BioScience $\mathbf{5 2}$ : 979-987.

Brown, J. H., S. K. M. Ernest, J. M. Parody, and J. P. Haskell. 2001. Regulation of diversity: maintenance of species richness in changing environments. Oecologia 126:321-332.

Brown, J. H., O. J. Reichman, and D. W. Davidson. 1979. Granivory in desert ecosystems. Annual Review of Ecology and Systematics 10:201-227.

Brown, J. H., T. J. Valone, and C. G. Curtin. 1997. Reorganization of an arid ecosystem in response to recent climate change. Proceedings of the National Academy of Sciences (USA) 94:9729-9733.

Collins, S. L. 2000. Disturbance frequency and community stability in native tall grass prairie. American Naturalist 155:311-325.

Collins, S. L., F. Micheli, and L. Hartt. 2000. A method to determine rates and patterns of variability in ecological communities. Oikos 91:285-293.

Currie, D. J. 1991. Energy and large-scale patterns of animalspecies and plant-species richness. American Naturalist 137:27-49.

Ernest, S. K. M., and J. H. Brown. 2001a. Homeostasis and compensation: the role of species and resources in ecosystem stability. Ecology 82:2118-2132.

Ernest, S. K. M., and J. H. Brown. 2001b. Delayed compensation for missing keystone species by colonization. Science 292:101-104.

Ernest, S. K. M., J. H. Brown, and R. R. Parmenter. 2000. Rodents, plants, and precipitation: spatial and temporal dynamics of consumers and resources. Oikos 88:470-482.

Guo, Q., J. H. Brown, and T. J. Valone. 2002. Long-term dynamics of winter and summer annual communities in the Chihuahuan Desert. Journal of Vegetation Science 13:575584.

Harvey, P. H., and H. C. J. Godfray. 1987. How species divide resources. American Naturalist 129:318-320.

Hawkins, B. A., R. Field, H. V. Cornell, D. J. Currie, J. F. Guegan, D. M. Kaufman, J. T. Kerr, G. G. Mittelbach, T. Oberdorff, E. M. O'Brien, E. E. Porter, and J. R. G. Turner. 2003. Energy, water, and broad-scale geographic patterns of species richness. Ecology 84:3105-3117.

Hubbell, S. P. 1979. Tree dispersion, abundance, and diversity in a tropical dry forest. Science 203:1299-1309.

Hubbell, S. P. 2001. The unified neutral theory of biodiversity and biogeography. Princeton University Press, Princeton, New Jersey, USA.

Hughes, J. B., and J. Roughgarden. 1998. Aggregate community properties and the strength of species interactions. Proceedings of the National Academy of Sciences (USA) 95:6837-6842.

Ives, A. R., K. Gross, and J. L. Klug. 1999. Stability and variability in competitive communities. Science 286:542544.
Kempton, R. A. 1979. The structure of species abundance and measurement of diversity. Biometrics 35:307-321.

Legendre, P., and L. Legendre. 1998. Numerical ecology. Elsevier Science, Amsterdam, The Netherlands.

MacArthur, R. H. 1955. Fluctuations of animal populations, and a measure of community stability. Ecology 36:533536.

MacArthur, R. H. 1957. On the relative abundance of bird species. Proceedings of the National Academy of Sciences (USA) 43:293-295.

Margalef, R. 1968. Perspectives in ecological theory. University of Chicago, Chicago, Illinois, USA.

Marquet, P. A., J. E. Keymer, and H. Cofre. 2003. Breaking the stick in space: of niche models, metacommunities, and patterns in relative abundance. Pages 64-84 in T. M. Blackburn and K. J. Gaston, editors. Macroecology: concepts and consequences. Blackwell Science, Oxford, UK.

May, R. M. 1973. Stability and complexity in model ecosystems. Princeton University Press, Princeton, New Jersey, USA.

May, R. M. 1975. Patterns of species abundance and diversity. Pages 81-120 in M. Cody and J. Diamond, editors. Ecology of species and communities. Harvard University Press, Cambridge, Massachusetts, USA.

McGowan, J. A., and P. W. Walker. 1985. Dominance and diversity maintenance in an oceanic ecosystem. Ecological Monographs 55:103-118.

Nagy, K., I. Girard, and T. Brown. 1999. Energetics of freeranging mammals, reptiles, and birds. Annual Review of Nutrition 19:247-277.

Pagel, M. D., P. H. Harvey, and H. C. J. Godfray. 1991. Species-abundance, biomass, and resource-use distributions. American Naturalist 138:836-850.

Preston, F. W. 1948. The commonness, and rarity, of species. Ecology 29:254-283.

Preston, F. W. 1962. The canonical distribution of commonness and rarity. I. Ecology 43:185-215.

Sugihara, G. 1980. Minimal community structure: an explanation of species abundance patterns. American Naturalist 116:770-787.

Sugihara, G., L. F. Bersier, T. R. E. Southwood, S. L. Pimm, and R. M. May. 2003. Predicted correspondence between species abundances and dendrograms of niche similarities. Proceedings of the National Academy of Sciences (USA) 100:5246-5251.

Taper, M. L., and P. A. Marquet. 1996. How do species really divide resources? American Naturalist 147:1072-1086.

Tokeshi, M. 1990. Niche apportionment or random assortment: species abundance patterns revisited. Journal of Animal Ecology 59:1129-1146.

Tokeshi, M. 1999. Species coexistence: ecological and evolutionary perspectives. Blackwell Science, Oxford, UK.

Valone, T. J., and J. H. Brown. 1995. Effects of competition, colonization, and extinction on rodent species diversity. Science 267:880-883.

White, E. P., S. K. M. Ernest, and K. M. Thibault. In press. Tradeoffs in community properties through time in a desert rodent community. American Naturalist.

Whittaker, R. H. 1965. Dominance and diversity in land plant communities. Science 147:250-260.

\section{APPENDIX}

Time series of standardized relative abundances and relative energy use within each rank of the granivorous rodent community, presented in figures and tables, are available in ESA's Electronic Data Archive: Ecological Archives E085-081-A1. 